\title{
国国pubvet
}

https://doi.org/10.31533/pubvet.v15n6a830.1-5

\section{Rotulagem de gelados comestíveis: avaliação da conformidade frente à legislação brasileira}

\author{
Márcia Rosália de Oliveira Sousa ${ }^{* \bullet}$, Aline Marques Monte ${ }^{2}$, Mariana Sousa Silva $^{3} \bullet$ \\ ${ }^{I}$ Tecnóloga em Gastronomia, Instituto Federal de Educação Ciência e Tecnologia do Piauí - IFPI, Teresina, PI Brasil. \\ ${ }^{2}$ Doutora em Alimentos e Nutrição, Universidade Federal do Piaui-UFPI, Teresina, PI Brasil. \\ ${ }^{3}$ Nutricionista, Universidade Federal do Piauí-UFPI, Teresina, PI Brasil. \\ *Autor para correspondências, E-mail: marcialeo2008@hotmail.com
}

Resumo. Os alimentos industrializados cada vez mais presentes na vida das pessoas têm em seu rótulo um elo de comunicação entre a indústria e o consumidor, que procuram por produtos adequados à sua condição de saúde. O objetivo deste estudo foi analisar se os rótulos de gelados comestíveis produzidos na cidade de Teresina-PI estão adequados as normas da Agência Nacional de Vigilância Sanitária. Foram analisados quanto a rotulagem, informação nutricional, uso de aditivos, informação quantitativa, peso líquido, unidade legal de massa, alimentos que causam alergia. Em todas as amostras há discordância com a Legislação. Faz-se necessário melhor controle e aplicação das normas por parte das indústrias e maior fiscalização governamental.

Palavras-chave: Gelados comestíveis, normas, rótulos

\section{Labeling of edible ice cream: evaluation of conformity against Brazilian legislation}

\begin{abstract}
Industrialized foods increasingly present in people's lives have on their label a communication link between the industry and the consumer, who look for products suitable to their health condition. The aim of this study was to analyze whether the labels of edible ice cream produced in the city of Teresina-PI are in line with the standards of the National Health Surveillance Agency. Were analyzed for labeling, nutritional information, use of additives, quantitative information, net weight, legal mass unit, foods that cause allergy. In all samples there is disagreement with the legislation. Better control and enforcement of standards by industries and greater government oversight are needed.
\end{abstract}

Keywords: edible ice cream, standards, labels

\section{Etiquetado de helados comestibles: evaluación de la conformidad con la legislación brasileña}

Resumen. Los alimentos industrializados cada vez más presentes en la vida de las personas tienen en su etiqueta un vínculo de comunicación entre la industria y el consumidor, que busca productos adecuados a su estado de salud. El objetivo de este estudio fue analizar si las etiquetas de los helados comestibles producidos en la ciudad de Teresina-PI se ajustan a los estándares de la Agencia Nacional de Vigilancia Sanitaria. Se analizaron por etiquetado, información nutricional, uso de aditivos, información cuantitativa, peso neto, unidad de masa legal, alimentos que causan alergia. En todas las muestras hay desacuerdo con la legislación. Se necesita un mejor control y aplicación de las normas por parte de las industrias y una mayor supervisión gubernamental.

Palabras clave: Helado comestible, estándares, etiquetas 


\section{Introdução}

Os alimentos industrializados cada vez mais presentes na vida das pessoas têm em seu rótulo um elo de comunicação entre a indústria e o consumidor, que procuram por produtos adequados à sua condição de saúde. A Lei 12.741/12 dispõe sobre as medidas de esclarecimentos ao consumidor e com base na Lei 8078/90 - Código de Proteção e Defesa do Consumidor no inciso III artigo $6^{\circ}$ garante a informação adequada e clara sobre os diferentes produtos e serviços, com especificação correta de quantidade, características, composição, qualidade, bem como sobre os riscos que apresentem (Cavalcante \& Holanda, 2017).

A legislação brasileira adota uma série de recomendações visando facilitar a leitura e compreensão das inscrições presentes nas embalagens, pois as mesmas servem de análise na hora da compra pelo consumidor, onde a capacidade e entendimento para interpretar às informações são necessários. A indústria por sua vez deve se adequar a estas normas com informações confiáveis, pois além de serem passíveis de multas e apreensões, coloca em risco a saúde dos consumidores (abris et al., 2006).

A RDC n ${ }^{\circ} 259 / 02$ define rotulagem como toda inscrição, legenda, imagem ou toda matéria descritiva ou gráfica, escrita, impressa, estampada, gravada, gravada em relevo ou litografada ou colada sobre a embalagem do alimento. Considera-se na Resolução $\mathrm{RDC} \mathrm{n}^{\circ} 360 / 03$ que a rotulagem nutricional é toda descrição destinada ao consumidor sobre as propriedades nutricionais de um alimento, compreendendo a declaração do valor energético e dos nutrientes, e também a declaração das propriedades nutricionais (Informação nutricional complementar). Com isso, a informação nutricional deve mencionar: valor energético, carboidratos, proteínas, gorduras totais, gorduras saturadas, gorduras trans, fibra alimentar e sódio, obedecendo à ordem aqui descrita (Baldani et al., 2018).

A informação nutricional deve corresponder ao valor encontrado em uma porção do alimento, que deve ser informada em grama $(\mathrm{g})$ ou mililitro $(\mathrm{mL})$, incluindo a medida caseira correspondente, segundo o estabelecido no regulamento técnico. Cada um dos itens deve também ser apresentado na forma de percentual de valores diários (\%VD). O padrão para o \%VD consiste numa dieta de $2000 \mathrm{kcal}$ ou 8400 $\mathrm{kJ}$, porém, deve conter a frase "a ingestão pode ser maior ou menor dependendo de suas necessidades energéticas" (Britto et al., 2016).

A RDC n ${ }^{\circ} 267$ de setembro de 2003 define como Gelados Comestíveis produtos alimentícios obtidos a partir de uma emulsão de gorduras e proteínas, com ou sem adição de outros ingredientes e substâncias, ou de uma mistura de água, açúcares e outros ingredientes e substâncias que tenham sido submetidas ao congelamento, em condições que garantam a conservação do produto no estado congelado ou parcialmente congelado, durante o armazenamento, o transporte, a comercialização e a entrega ao consumo (Jardim et al., 2017).

O objetivo deste estudo é analisar se os rótulos de Gelados Comestíveis produzidos na cidade de Teresina-PI estão em conformidade com a legislação brasileira.

\section{Material e métodos}

Para análise dos rótulos foram utilizadas as Resoluções demonstradas no quadro abaixo. Verificouse em cada rótulo dos gelados comestíveis a concordância dos termos usados de acordo com as resoluções e portarias constantes no quadro 1.

Analisou-se três marcas de produtos, sendo dois rótulos de cada de sabores diferentes. As amostras foram adquiridas em supermercados e farmácias na cidade de Teresina no mês de fevereiro de 2019 e possuem registro para comercialização. 
Quadro 1. Legislações usadas para análise de rótulos das amostras. Teresina, 2019.

\begin{tabular}{|c|c|c|}
\hline Assunto & Legislação & Especificação \\
\hline Atribuição de Aditivos & $\begin{array}{l}\text { RDC n' 3/2007 } \\
\text { (Brasil. ANVISA, 2007) }\end{array}$ & $\begin{array}{l}\text { Estabelece limites máximos para o uso de aditivos em } \\
\text { alimentos. }\end{array}$ \\
\hline Rotulagem de alimentos embalados & $\begin{array}{l}\mathrm{RDC} \mathrm{n}^{\circ} \text { 259/2002 } \\
\text { (Brasil. ANVISA, 2002) }\end{array}$ & $\begin{array}{l}\text { Trata do Regulamento Técnico sobre Rotulagem de } \\
\text { Alimentos Embalados. }\end{array}$ \\
\hline Rotulagem nutricional & $\begin{array}{l}\mathrm{RDC} \mathrm{n}^{\circ} \text { 360/2003 } \\
\text { (Brasil. ANVISA, } 2003\end{array}$ & $\begin{array}{l}\text { Dispõe do Regulamento Técnico sobre Rotulagem } \\
\text { Nutricional de Alimentos Embalados. }\end{array}$ \\
\hline Medidas e porções & $\begin{array}{l}\mathrm{RDC} \mathrm{n}{ }^{\circ} 359 / 2003 \\
\text { (Brasil. ANVISA, 2003) }\end{array}$ & $\begin{array}{l}\text { Trata do Regulamento Técnico de porções de } \\
\text { Alimentos para fins de Rotulagem Nutricional. }\end{array}$ \\
\hline Informação Nutricional Complementar & $\begin{array}{l}\mathrm{RDC} \mathrm{n}^{\circ} 54 / 2012 \\
\text { (Brasil. ANVISA, 2012) }\end{array}$ & $\begin{array}{l}\text { Estabelece os termos usados para a Informação } \\
\text { Nutricional Complementar. }\end{array}$ \\
\hline Expressa a indicação quantitativa & $\begin{array}{l}\text { Portaria } \mathrm{n}^{\circ} 157 / 2002 \\
\text { (Brasil. INMETRO, 2002) }\end{array}$ & $\begin{array}{l}\text { Estabelece a forma de expressar a indicação quantitativa } \\
\text { do conteúdo líquido dos produtos pré-medidos. }\end{array}$ \\
\hline Indica o volume e a massa do produto & $\begin{array}{l}\text { Portaria } \mathrm{n}^{\circ} 077 / 2007 \\
\text { (Brasil. INMETRO, 2007) }\end{array}$ & $\begin{array}{l}\text { Dispõe que sorvetes pré-medidos devem indicar o } \\
\text { conteúdo líquido e em unidade legal de massa. }\end{array}$ \\
\hline Alimentos alergênicos & $\begin{array}{l}\text { RDC n }{ }^{\circ} 26 / 2015 \\
\text { (Brasil. ANVISA, 2015) }\end{array}$ & $\begin{array}{l}\text { Estabelece os requisitos para rotulagem obrigatória } \\
\text { dos principais alimentos que causam alergia. }\end{array}$ \\
\hline Gelados comestíveis & $\begin{array}{l}\text { RDC } n^{\circ} 266 / 2005 \\
\text { (Brasil. ANVISA, 2005) }\end{array}$ & $\begin{array}{l}\text { Regulamento Técnico para Gelados Comestíveis e } \\
\text { preparados para Gelados Comestíveis. }\end{array}$ \\
\hline Gelados comestíveis & $\begin{array}{l}\mathrm{RDC}^{\circ} \text { 267/2003 } \\
\text { (Brasil. ANVISA, 2003) }\end{array}$ & $\begin{array}{l}\text { Regulamento Técnico de Boas Práticas de Fabricação } \\
\text { para Gelados Comestíveis. }\end{array}$ \\
\hline
\end{tabular}

\section{Resultados e discussão}

Todas as marcas pesquisadas possuem rótulos, dados do fabricante, denominação de venda, lista de ingredientes, conteúdo líquido, informação nutricional, porção e medida caseira, aditivos permitidos.

Não conformidades

Dentre as não conformidades, de acordo com as Resoluções constantes no quadro 1, destaca-se:

Lote - ausência deste número que serve de rastreamento no caso do alimento com irregularidade, não encontrado em duas das três marcas analisadas;

Modo de conservação - há discordância entre as marcas analisadas sobre este item, dois produtos da mesma marca descrevem condições de armazenamento em temperaturas diferentes. Na terceira, a temperatura está igual em ambos os produtos, porém em desacordo com a legislação.

Legibilidade dos textos - um dos rótulos analisados não possui o tamanho da letra adequada, e a cor não contrasta com a cor do fundo do rótulo, causando desconforto visual.

Valores diários de referência - o valor de referência diário de ingestão de calorias está com 2,500 calorias, em uma das marcas consultadas, o que está em desacordo com a norma vigente.

Porção e medida caseira - uma marca apresenta a porção em desacordo com a norma.

Alimentos alergênicos - dois rótulos não apresentam informação sobre alimentos alergênicos e as expressões como "contém glúten" ou "não contém glúten" que servem para advertir, prevenir ou controlar a doença celíaca não estando presentes.

\section{Discussão}

A rotulagem é fundamental para a saúde pública e as informações contidas nos rótulos são muito importantes, pois possibilitam identificar a origem do produto, composição, características nutricionais, garantindo aos consumidores uma informação útil e confiável (Lagerkvist, 2013).

Não deve servir apenas à propaganda do produto, respeitar as normas, ser fiel ao alimento e ao consumidor, com informações claras e precisas. Yoshizawa et al. (2003) concluíram que a maioria dos rótulos dos produtos alimentícios comercializados apresentam irregularidades em relação à legislação vigente, incluindo-se a ausência de declarações e/ou advertências obrigatórias, onde a aquisição de produtos é influenciada por estratégias de mercado. 
O governo estabelece critérios para normatizar a rotulagem, mas é ineficiente na fiscalização, há ausência de declarações e advertências essenciais previstas em lei e necessárias para orientar o consumidor. Pontes et al. (2009) citam que dados recentes demonstram que 70\% das pessoas consultam rótulos dos alimentos no momento da compra; no entanto, mais da metade não compreende adequadamente o significado das informações. Outra pesquisa, realizada em Brasília, confirma esse resultado: $74,8 \%$ dos pesquisados leem as informações nutricionais, embora apenas $25 \%$ desse grupo tenha o hábito de ler os rótulos de todos os alimentos. Mais da metade desses consumidores que consultava os rótulos lia apenas os referentes a alimentos específicos, com o objetivo de conhecer seu valor calórico. Os resultados obtidos em ambas as pesquisas indicaram que, apesar de a população considerar importante que o rótulo de alimentos contenha as informações nutricionais, a maioria não sabe utilizá-los.

O consumidor brasileiro deve saber o que consome. Em produtos industrializados essa informação está no rótulo (Netto et al., 2018), mas não há conhecimento necessário para interpretar essas informações, ainda que muitas vezes não corretamente escrita. Importante uma educação nutricional que $o$ ajude a entender.

\section{Conclusões}

Todos os rótulos analisados apresentaram divergência na aplicação da legislação. Faz-se necessário maior compromisso por parte das empresas em apresentar informações fiéis à legislação brasileira para assim garantirem a segurança de quem consome seus produtos. Quanto aos consumidores também é importante que saibam identificar corretamente o que estão consumindo, pois a grande maioria lê o rótulo, mas não sabe o significado principalmente da informação nutricional. Por parte do governo é necessário maior controle nas indústrias produtoras de gelados comestíveis e disseminar o conhecimento nutricional.

\section{Referências}

Baldani, M. M., Pascoal, G. B., \& Rinaldi, A. E. M. (2018). Labeling and commercial promotion of infant formulas retailed in Brazil. DEMETRA: Food, Nutrition \& Health/Alimentação, Nutrição \& Saúde, 13(2), 413-425.

Brasil. (2002). Resolução RDC n ${ }^{\circ} 259$, de 20 de setembro de 2002. Regulamento Técnico sobre Rotulagem de Alimentos Embalados. Diário Oficial da União. Recovered on Feb. 05, 2020 from: http://www.agricultura.gov.br/assuntos/inspecao/produtos-vegetal/legislacao-1/biblioteca-denormas-vinhos-e-bebidas/resolucao-rdc-no-259-de-20-de-setembro-de-2002.pdf/view

Brasil. (2003) a. Resolução RDC n ${ }^{\circ}$ 359, de 23 de setembro de 2003. Regulamento Técnico de Porções de Alimentos Embalados para Fins de Rotulagem Nutricional. Diário Oficial da União. Recovered on Feb. $\quad 05, \quad 2020$ from: http://portal.anvisa.gov.br/documents/33880/2568070/res0359_23_12_2003.pdf/76676765-a10740d9-bb34-5f05ae897bf3

Brasil. (2003) b. Resolução RDC ANVISA n 267, de 25 de setembro de 2003. Regulamento Técnico para Estabelecimentos Industrializadores de Gelados Comestíveis. Diário Oficial da União. Recovered on $\quad$ Feb. $05, \quad 2020$ from: http://portal.anvisa.gov.br/documents/33916/388704/RDC_N_267.pdf/6bbd5fab-2c85-4b80-9c0b1 ad6ea $42 \mathrm{~d} 5 \mathrm{c} 0$

Brasil. (2003) c. Resolução RDC n 360, de 23 de dezembro de 2003. Regulamento Técnico sobre Rotulagem Nutricional de Alimentos. Diário Oficial da União. Recovered on Feb. 05, 2020 from: http://portal.anvisa.gov.br/documents/33880/2568070/res0360_23_12_2003.pdf/5d4fc713-9c664512-b3c1-afee57e7d9bc

Brasil. (2005). Resolução RDC n 266, de 22 de setembro de 2005. Regulamento Técnico para Gelados Comestíveis e Preparados para Gelados Comestíveis. Diário Oficial da União. Recovered on Feb. 05, $2020 \quad$ from: http://portal.anvisa.gov.br/documents/10181/4188550/RDC_266_2019_.pdf/2fe7c084-24db-48de8bc6-33c9c6481d7b 
Brasil. (2007). Resolução RDC n 3, de 15 de janeiro de 2007. Regulamento Técnico sobre Atribuição de Aditivos e Seus Limites pra a Categoria de Alimentos 3: Gelados Comestíveis. Diário Oficial da União. Recovered on Feb. $05, \quad 2020$ from: http://portal.anvisa.gov.br/documents/33916/391619/Resolu\%25C3\%25A7\%25C3\%25A3o\%2BR DC\%2Bn\%25C2\%25BA\%2B3\%252C\%2Bde\%2B15\%2Bde\%2Bjaneiro\%2Bde\%2B2007.pdf/807 3d0d6-fe22-4af8-99d6-fe0788a91448

Brasil. (2012). Resolução RDC no 54, de 12 de novembro de 2012. Regulamento Técnico sobre Informação Nutricional Complementar. Diário Oficial da União. Recovered on Feb. 05, 2020 from: http://portal.anvisa.gov.br/documents/\%2033880/2568070/rdc0054_12_11_2012.pdf/c5ac23fd974e-4f2c-9fbc-48f7e0a31864

Brasil. (2015). Resolução RDC nº 26, de 02 de julho de 2015. Regulamento Técnico sobre os Requisitos para Rotulagem Obrigatória dos Principais Alimentos que causam Alergias Alimentares. Diário Oficial da União. Recovered on Feb. 05, 2020 from: http://www.agricultura.gov.br/assuntos/inspecao/produtos-vegetal/legislacao-1/biblioteca-denormas-vinhos-e-bebidas/resolucao-rdc-no-26-de-2-de-julho-de-2015.pdf/view.

Britto, L. F., Silva, A. de P. V., Mendes, L. G., \& Medeiros, S. R. A. (2016). Avaliação da rotulagem de alimentos à base de cereais para a alimentação de lactentes e crianças na primeira infância. DEMETRA: Food, Nutrition \& Health/Alimentação, Nutrição \& Saúde, 11(1), 111-120.

Cavalcante, D. L., \& Holanda, F. C. C. (2017). Relações de consumo e transparência fiscal: o descaso em relação a lei n. 12.741/2012. Revista de Direito Internacional Econômico e Tributário, 12(1 Jan/Jun), 246-260.

Fabris, S., Freire, M. T. A., \& Reyes, F. G. R. (2006). Embalagens plásticas: tipos de materiais, contaminação de alimentos e aspectos de legislação. Revista Brasileira de Toxicologia, 19(2), 5970.

Jardim, F. B. B., Damasceno, K. A., Pereira, L. M., Santos, L. C. R., Vilela, L. R., Carneiro, I. M. M., Oliveira, M., Cunha, G. R. R., \& da Silva, A. C. (2017). Boas práticas de fabricação para estabelecimentos de gelados comestíveis. Boletim Técnico IFTM, 5, 10-15.

Lagerkvist, C. J. (2013). Consumer preferences for food labelling attributes: Comparing direct ranking and best-worst scaling for measurement of attribute importance, preference intensity and attribute dominance. Food Quality and Preference, 29(2), 77-88. https://doi.org/http://dx.doi.org/10.1016/j.foodqual.2013.02.005

Netto, L. B. C., Silva, L. M., \& Xavier, M. M. B. B. S. (2018). Qualidade e rotulagem de ovos comercializados no município de Valença-RJ. PUBVET, 12(9), 1-9. https://doi.org/http://dx.doi.org/10.31533/pubvet.v12n9a173.1-9.

Pontes, T. E., Costa, T. F., Marum, A. B. R. F., Brasil, A. L. D., \& Taddei, J. A. A. C. (2009). Orientação nutricional de crianças e adolescentes e os novos padrões de consumo: propagandas, embalagens e rótulos. Revista Paulista de Pediatria, 27(1), 99-105.

Yoshizawa, N., Pospissil, R. T., Valentim, A. G., Seixas, D., Alves, F. S., Cassou, F., Yoshida, I., SEGA, R. A., \& Cândido, L. M. B. (2003). Rotulagem de alimentos como veículo de informação ao consumidor: adequações e irregularidades. Boletim Do Centro de Pesquisa de Processamento de Alimentos, 21(1), 169-180.

Histórico do artigo:

Recebido: 2 de novembro de 2020.

Aprovado: 15 de dezembro de 2020.
Licenciamento: Este artigo é publicado na modalidade Acesso Aberto sob a licença Creative Commons Atribuição 4.0 (CC-BY 4.0), a qual permite uso irrestrito, distribuição, reprodução em qualquer meio, desde que o autor e a fonte sejam devidamente creditados. 